\title{
Distribution of Malassezia Species in Patients with Pityriasis versicolor Compared with Healthy Individuals in Yazd, Iran
}

\author{
Abbas Ali Jafari ${ }^{1}$, Hossein Zarrinfar ${ }^{2,3}$, Farzanenh Mirzaei ${ }^{1}$, Farzad Katiraee ${ }^{4,}$ \\ ${ }_{1}^{1}$ Medical Parasitology and Mycology Department, Medical School, Shahid Sadoghi University of Yazd Medical Sciences, Yazd, IR Iran \\ 2 Department of Medical Parasitology and Mycology, Ghaem Hospital, School of Medicine, Mashhad University of Medical Sciences, Mashhad, IR Iran \\ 3 Allergy Research Center, Mashhad University of Medical Sciences, Mashhad, IR Iran \\ 4 Allergy Research Center, Mashhad University of Medical Sciences, Mashhad, IR Iran \\ ${ }^{*}$ Corresponding author: Farzad Katiraee, Department of Pathobiology, Faulty of Veterinary Medicine, University of Tabriz, Tabriz, IR Iran. Tel:+98-9126047311, Fax: +984116378743, E- \\ mail: katiraee_f@yahoo.com.
}

Received: Jun 21, 2012; Revised: September 30, 2012; Accepted: October 03, 2012

\begin{abstract}
Background: Malassezia species as skin microflora of humans and other warm-blooded vertebrates are the lipophilic yeasts associated with various human diseases, especially pityriasis versicolor(PV).

Objectives:The objective of this studywas toidentify the Malassezia species of scraped skin of PV patients in Yazd, Iran, using morphological, biochemical and physiological methods. We also compared the obtained results of PV patients with normal healthy volunteers.

Materials and Methods: $A$ total of 200 persons, including 100 patients (with skin lesion) referred to Yazd Central Laboratory and 100 healthy volunteers as controls, were evaluated for Malassezia infection by morphological and biochemical methods.

Results: The most commonly isolated species from PV lesions, were M. globosa (38.3\%), M. furfur (29.4\%), M. sympodialis (14.9\%), M. pachydermatis (9.6\%) and M. slooffiae (5.3\%). Also the most commonly isolated species from healthy skins were M. furfur (37.2\%), M. globosa (25.6\%), M. sympodialis (16.3\%), M. pachydermatis (13.9\%) and M. slooffiae (4.6\%). Totally M. globosa and M. furfur were the most frequented isolated.

Conclusion: Highest prevalence of PV in our study was observed in the 20-39 years old group, suggesting that the peak of the infection is coincided with ages and increasing sebum production in the highest level. M. globosa was the most commonly isolated Malassezia species of the patients group and $M$. furfur is the most common isolated species obtained from normal individuals skin samples. We couldn't find any significant differences between groups. The rate of isolated Malassezia species from patients was higher than normal individuals.
\end{abstract}

Keywords: Malassezia; Pityriasis versicolor, Malassezia globosa; Iran

\section{Background}

Malassezia species are lipophilic yeasts and parts of skin microflora of humans and other warm-blooded vertebrates that associated with various human diseases, especially pityriasis versicolor (PV), a chronic superficial scaling dermatomycosis (1-3). This chronic superficial fungal disease is characterized by the appearance of round-tooval lesions. This disease is common in young adults and usually presented as variable pigmented scaling macules (3). These lesions vary in color, and can be hypo or hyperpigmented. The most common sites of disease are the upper trunk and the neck (4). Numerous studies have shown the relationship between Malassezia species and their role as causative agents and/or triggers of diseases. Also Malassezia species, are etiological agents of catheterassociated fungemia, seborrheic dermatitis (5), and folliculitis $(3,6)$ dacryolitis, blepharitis as well as nosoco- mial bloodstream infections in pediatric care units $(7,8)$. The taxonomy of genus Malassezia was controversial and has undergone several taxonomic revisions. In 1996, in the reclassification by Gueho et al. seven distinct species were identified within this genus namely $M$. furfur, $M$. pachydermatis, M. sympodialis, M. globosa, M. obtusa, M. restricta and M. slooffiae. Recently, on the basis of DNA relatedness, six new species have been included in this genus: M. dermatis, M. nana, M. japonica, and M. yamatoensis, $M$. equine, $M$. caprae (6, 9-14). Among them, M. restricta and $M$. globosa are considered to be as the most important pathogenic organisms in the development of seborrheic dermatitis. However, some reports have also linked M. furfur, M. sympodialis, M. obtusa, and M. slooffiae with seborrheic dermatitis. Our data about the epidemiology of PV in Iran is limited in some regions such as Tehran, Kashan, Ahvaz and Mazandaran (6,15-18). Also there are evidences suggesting that the geographical variations of the spe- 
cies are available.

\section{Objectives}

The objective of this study was to identify the Malassezia species on the scalp of patients with PV, in Yazd (a central province in Iran) using morphological, biochemical and physiological modifying methods. We also compared the results of PV patients with normal healthy volunteers.

\section{Patients and Methods}

\subsection{Subjects and Collecting Samples}

A total of 200 samples, including 100 patients (with skin lesion) referred to Yazd Central Laboratory and 100 healthy volunteers as control group were subjected to this study (Table 1). A questioner included, gender, age, duration of disease, lesion type and involved area were filled for each person. Initially, PV on the lesions was clinically diagnosed, after that the final diagnosis was confirmed by direct microscopic examination with Sellotape method and methylene blue staining of the samples collected from scraped skin of patients (19 - 21). In control group, scraped skin was collected as same as patients, from chest, back, arms, face and neck and abdominal area.

\begin{tabular}{|c|c|c|c|c|c|c|}
\hline \multirow[t]{2}{*}{ Age Groups } & \multicolumn{3}{|c|}{ Pityriasis versicolor } & \multicolumn{3}{|c|}{ Control Group } \\
\hline & Male, No. (\%) & Female, No. (\%) & Total, \% & Male, No. (\%) & Female, No. (\%) & Total, \% \\
\hline$<19, y$ & $14(25.9)$ & $8(17.4)$ & 22 & $11(21.6)$ & $9(18.3)$ & 20 \\
\hline $20-39, y$ & $21(38.9)$ & $19(41.3)$ & 40 & $23(45.1)$ & $22(44.9)$ & 45 \\
\hline $40-59, y$ & $15(27.8)$ & $13(28.2)$ & 28 & $12(23.5)$ & $14(28.6)$ & 26 \\
\hline$>60, y$ & $4(7.4)$ & $6(13.1)$ & 10 & $5(9.8)$ & $4(8.2)$ & 9 \\
\hline Total & $54(100)$ & $46(100)$ & 100 & $51(100)$ & $49(100)$ & 100 \\
\hline
\end{tabular}

\subsection{Culture of Samples}

The scraped skin was inoculated in plates containing modified Dixon medium (Merck, Germany) (mDixon). This medium consisted of 3.6\% malt extract, $2.0 \%$ desiccated Ox-bile, $1.0 \%$ Tween $40,0.2 \%$ glycerol, $0.2 \%$ oleic acid, $0.05 \%$ chloramphenicol, $0.5 \%$ cycloheximide, and $1.2 \%$ agar. Inoculated cultures incubated at $32^{\circ} \mathrm{C}$ for $1-10$ days. According to Gueho et al. and Mayer et al. suspected Malassezia spp. identified on the basis of the colony morphology, microscopic appearance of the fungal cells obtained in the culture, by examining the fungal ability to grow without lipid supplementation $(13,22)$.

\subsection{Tween Assimilation Test}

Utilization of different Tween compounds (Tween 20, 40,60 and 80)(Sigma-Aldrich, USA) as a lipid supplement for Malassezia species performed According to the Guillot et al. (20) and Gupta et al. (3), Briefly, the yeast suspension (at least $10^{5}$ to $2 \times 10^{5} \mathrm{CFU} / \mathrm{mL}$ ) was made in $2 \mathrm{~mL}$ sterilized distilled water and poured into plate containing Sabouraud dextrose agar (Merck, Germany) at $45^{\circ} \mathrm{C}$. The inoculums were then spread evenly. After solidification of each plate, four wells with $2 \mathrm{~mm}$ diameter were made and filled with $10 \mu \mathrm{L}$ Tween 20,40, 60 and 80, respectively. These plates were incubated for a week at $31^{\circ} \mathrm{C}$ and the growing degree was assessed by measuring the sedimentation zone of each well after 2, 4 and 7 days $(21,23)$.

\subsection{Catalase Reaction}

Presence of catalase was determined by using a drop of hydrogen peroxide (3\% solution) and production of gas bubbles that considered as a positive reaction. Lack of catalase activity is a characteristic feature of M. restricta, $(20,21)$ and also M. pachydermatis is variable in catalase reaction (21).

\subsection{Splitting of Esculin}

The $\beta$-glucosidase activity of different Malassezia species was assayed using method described by Mayser et al. (22). Briefly, a loop of fresh yeasts was inoculated deeply in the esculin agar (Merck, Germany) tube and incubated for 5 days at $31^{\circ} \mathrm{C}$. The splitting of esculin is revealed by darkening of the medium. This test was used to differentiate $M$. furfur, M. slooffiae and M. sympodialis from other Malassezia species. However M. pachydermatis has a variable reaction (1). In order to differentiation of $M$. sympodialis and M. obtusa, they were incubated at $40^{\circ} \mathrm{C}$ for one week $(1,13)$.

\subsection{Demonstration of M. pachydermatis}

As M. pachydermatis is the only non-obligatory lipid dependent species of Malassezia spp. $(21,24)$, the yeast isolated on mDixon agar was smeared with a sterile swab on Sabouraud glucose agar (Merck, Germany) plus 0.05\% chloramphenicol and $0.05 \%$ cyclohexamiede devoid of lipids. Incubation was performed at $31^{\circ} \mathrm{C}$ for one week.

\subsection{Statistical Analysis}

Quantitative data were analyzed by t-test. The data of the patient and healthy controls were analyzed using chisquare test and Fisher exact test. P-values of $<0.05$ were 
considered significant.

\section{Results}

The study included 100 patients (46 women and 54 men) with PV, aged $18-72$ and 100 healthy individuals aged 19 - 70 years. No statistically significant difference was observed in the frequency of isolated Malassezia spp. between women and men within group or between groups (P: 0.34). The highest prevalence of PV was observed in patients with 20 - 39 years old. Significant differences in the rate of Malassezia species isolation between age groups were observed in both groups $(\mathrm{P}<0.04) .94 \%$ of the specimens in patients with $\mathrm{PV}$ and $43 \%$ of healthy individuals yielded Malassezia in culture. Besides, culture positive cases were higher in patient group than healthy controls and this difference was statistically significant $(\mathrm{P}<0.05)$.

In PV lesions, the most commonly isolated species was M. globosa (38.3\%), M. furfur (29.4\%), M. sympodialis (14.9\%), M. pachydermatis (9.6\%) and M. slooffiae (5.3\%), respectively. the most commonly isolated species were $M$. furfur (37.2\%), M. globosa (25.6\%), M. sympodialis (16.3\%), M. pachydermatis (13.9\%) and M. slooffiae (4.6\%), respectively. Totally M. globosa and M. furfur were the frequent isolations. Overall, no differences in distribution of Malassezia isolated species were noted in 2 groups $(\mathrm{P}=0.1)$. However in patient's group isolation of M. globosa was higher than M. furfur . Predominantly, Malassezia species were isolated from the chest. Overall in patients group, 26 isolates were obtained from this organ. On the other hand, in healthy individuals Malassezia species predominantly were obtained from back and chest area (13 and 12 cases respectively). Also the lowest isolation of Malassezia species was from arm in both groups (Figure 1). Tables 1 and 2; show the distribution of subjects and Malassezia species, based on the sites of sample collection.

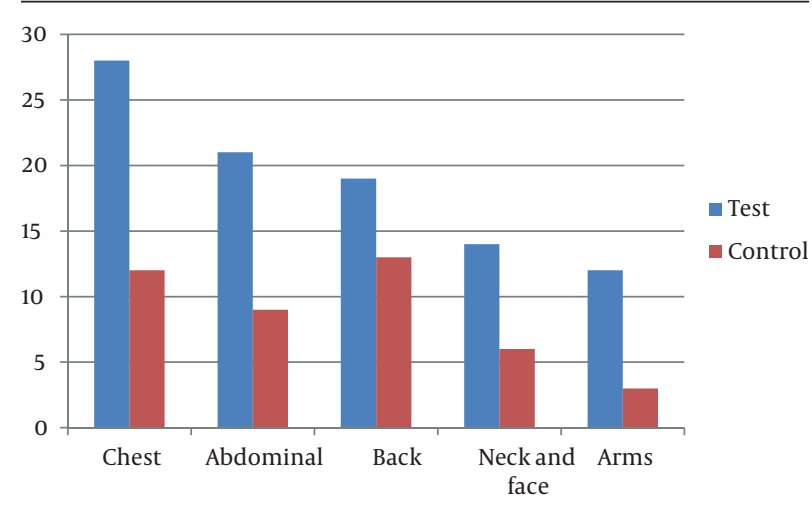

Figure 1. Distribution of Malassezia Isolated Based on Anatomical Sites

Table 2. Distribution of Different Malassezia Species Isolated in Patients and Healthy Individual

\begin{tabular}{|c|c|c|c|c|c|c|c|}
\hline \multirow[t]{2}{*}{ Species } & \multicolumn{3}{|c|}{ Patients } & \multicolumn{3}{|c|}{ Normal } & \multirow[t]{2}{*}{ Total, No. (\%) } \\
\hline & Male, \% & Female, \% & Total, No. (\%) & Male, \% & Female, \% & Total, No. (\%) & \\
\hline M. globosa & 22 & 14 & $36(38.3)$ & 6 & 5 & $11(25.6)$ & $47(34.3)$ \\
\hline M. furfur & 15 & 12 & $27(29.4)$ & 9 & 7 & $16(37.2)$ & $42(30.6)$ \\
\hline M. sympodialis & 4 & 10 & $14(14.9)$ & 3 & 4 & $7(16.3)$ & $21(15.3)$ \\
\hline M.pachydermatis & 6 & 3 & $9(9.6)$ & 4 & 2 & $6(13.9)$ & $15(10.9)$ \\
\hline M. slooffiae & 2 & 3 & $5(5.3)$ & 2 & 0 & $2(4.6)$ & $7(5.2)$ \\
\hline Malassezia.Species & 1 & 2 & $3(3.2)$ & 1 & 0 & $1(2.3)$ & $4(2.9)$ \\
\hline Total & 50 & 44 & $94(100)$ & 25 & 18 & $43(100)$ & $137(100)$ \\
\hline
\end{tabular}

\section{Discussion}

Different studies showed that the frequency and occurrence of Malassezia species and their diseases is depending on some factors such as occupational and economical conditions as well as climate and geographical area $(6,18,25)$. In many studies $M$. globosa is the predominant species in lesional and normal skin. In our study similar to other investigations, in PV patients M. globosa was the predominant species, but in normal individual M. furfur was the most isolated species. On the other hand, similar to other investigations the highest prevalence of PV in our study was observed in 20 - 39 years old group, suggesting that the high infection is associated with the age and increasing sebum production at the highest level (6,
17, 26). Previous reports showed that PV is uncommon among children (6) and we didn't found any case of PV in children. However it is only rarely found in the elderly, we had more cases of PV in over 60 years old individuals. In addition, $M$. sympodialis was isolated more frequently than what has been reported by many Iranian investigators expressing that this species is known as the third or fourth frequented species $(6,15,17,21)$. We couldn't isolate any M. obtusa and M. restricta in this study, although in other studies in Iran these species were isolated in at a very low frequency. $M$. slooffiae is less common in other studies and we isolated this species in 5.3\% of patients and $4.6 \%$ in normal individuals. Interestingly, M. pachydermatis was isolated in $9.6 \%$ and $13.0 \%$ of patients and healthy individuals respectively, however this species is 
associated with animals.

In other reports from Iran, M. pachydermatis was not isolated or low frequency isolated (21). These differences between our study and others may be due to geographical variation and some laboratory techniques such as sampling and differentiation methods. We have isolated a single colony from each sample collected from patients with PV, as suggested by some investigators. However, in many studies, more than one species has been recovered from each sample group. It should be underlined that providing a pure culture and the isolation of a species from a mixed culture is too difficult. This might be due to this point that fast growing species usually cover other species in the culture.

All pathogenic species in patients group were detected in normal individual that showed PV is caused as a secondary complication for patients that is not usually pathogenic. In conclusion, we compared the recovery rate of Malassezia species on the lesions of patients with PV and skin of normal persons. M. globosa was the most commonly isolated species in the patients group and $M$. furfur in normal individuals. However we couldn't find any significant differences of Malassezia species distribution between groups. In addition, the rate of isolation of Malassezia species in patients was higher than normal individuals.

\section{Acknowledgements}

This study was financially supported by Shahid Sadoughi University of Yazd Medical Sciences. We express our appreciation to Dr. Fallah Zadeh for his help in the statistical analysis.

\section{Authors' Contribution}

None declared.

\section{Financial Disclosure}

None declared.

\section{Funding/Support}

None declared.

\section{References}

1. Midgley G. The diversity of Pityrosporum (Malassezia) yeasts in vivo and in vitro. Mycopathologia.1989;106(3):143-53.

2. Leeming JP, Notman FH, Holland KT. The distribution and ecology of Malassezia furfur and cutaneous bacteria on human skin. J Appl Bacteriol. 1989;67(1):47-52.

3. Gupta AK, Bluhm R, Summerbell R. Pityriasis versicolor. J Eur Acad Dermatol Venereol. 2002;16(1):19-33.

4. Crespo Erchiga V, Delgado Florencio V. Malassezia species in skin diseases. Curr Opin Infect Dis. 2002;15(2):133-42.

5. DeAngelis YM, Gemmer CM, Kaczvinsky JR, Kenneally DC, Schwartz JR, Dawson TL, Jr. Three etiologic facets of dandruff and seborrheic dermatitis: Malassezia fungi, sebaceous lipids, and individual sensitivity. J Investig Dermatol Symp Proc.
2005;10(3):295-7

6. Tarazooie B, Kordbacheh P, Zaini F, Zomorodian K, Saadat F, Zeraati H, et al. Study of the distribution of Malassezia species in patients with pityriasis versicolor and healthy individuals in Tehran, Iran. BMC Dermatol. 2004;4:5.

7. Chryssanthou E, Broberger U, Petrini B. Malassezia pachydermatis fungaemia in a neonatal intensive care unit. Acta Paediatr 2001;90(3):323-7.

8. Gaitanis G, Magiatis P, Hantschke M, Bassukas ID, Velegraki A. The Malassezia genus in skin and systemic diseases. Clin Microbiol Rev. 2012;25(1):106-41.

9. Sugita T, Takashima M, Shinoda T, Suto H, Unno T, Tsuboi R, et al. New yeast species, Malassezia dermatis, isolated from patients with atopic dermatitis. JClin Microbiol. 2002;40(4):1363-7.

10. Sugita T, Kodama M, Saito M, Ito T, Kato Y, Tsuboi R, et al. Sequence diversity of the intergenic spacer region of the rRNA gene of Malassezia globosa colonizing the skin of patients with atopic dermatitis and healthy individuals. J Clin Microbiol. 2003;41(7):30227.

11. Sugita T, Tajima M, Takashima M, Amaya M, Saito M, Tsuboi R, et al. A new yeast, Malassezia yamatoensis, isolated from a patient with seborrheic dermatitis, and its distribution in patients and healthy subjects. Microbiol Immunol. 2004;48(8):579-83.

12. Hirai A, Kano R, Makimura K, Duarte ER, Hamdan JS, Lachance MA, et al. Malassezia nana sp. nov., a novel lipid-dependent yeast species isolated from animals. Int J Syst Evol Microbiol. 2004;54(Pt 2):623-7.

13. Gueho E, Midgley G, Guillot I. The genus Malassezia with description of four new species. Antonie Van Leeuwenhoek. 1996;69(4):337-55.

14. Sei Y. [Malassezia infections]. Med Mycol J. 2012;53(1):7-11.

15. Zomorodian K, Mirhendi H, Tarazooie B, Zeraati H, Hallaji Z, Balighi K. Distribution of Malassezia species in patients with psoriasis and healthy individuals in Tehran, Iran.J Cutan Pathol. 2008;35(11):1027-31.

16. Shokohi T, Afshar P, Barzgar A. Distribution of Malassezia species in patients with pityriasis versicolor in Northern Iran. Indian J Med Microbiol. 2009;27(4):321-4.

17. Nazeri M, Amiri S, Moniri R, Moayeri MR, Morveji AR, Asghari B, et al. Isolation and identification of Malassezia spp. in pityriasis versicolor patients in Kashan. Kowsar Med J. 2009;13(4):293-296.

18. Mahmoudabadi AZ, Zarrin M. Pityriasis versicolor in Ahvaz, Iran. Jundishapur J Microbiol. 2009;2(3):92-96.

19. Hedayati MT, Hajheydari Z, Hajjar F, Ehsani A, Shokohi T, Mohammadpour R. Identification of Malassezia species isolated from Iranian seborrhoeic dermatitis patients. Eur Rev Med Pharmacol Sci. 2010;14(1):63-8.

20. Guillot J, Guého E, Lesourd M, Midgley G, Chévrier G, Dupont B. Identification of Malassezia species: a practical approach. $J$ de mycologie médicale. 1996;6(3):103-110.

21. Khosravi AR, Eidi S, Katiraee F, Ziglari T, Bayat M, Nissiani M. Identification of different Malassezia species isolated from patients with Malassezia infections. World JZoology. 2009;4(2):85-89.

22. Mayser P, Wille G, Imkampe A, Thoma W, Arnold N, Monsees T. Synthesis of fluorochromes and pigments in Malassezia furfur by use of tryptophan as the single nitrogen source. Mycoses. 1998;41(7-8):265-71.

23. Guillot J, Hadina S, Gueho E. The genus Malassezia: old facts and new concepts. Parassitologia. 2008;50(1-2):77-9.

24. Raabe P, Mayser P, Weiss R. Demonstration of Malassezia furfur and M. sympodialis together with M. pachydermatis in veterinary specimens. Mycoses. 1998;41(11-12):493-500.

25. Gaitanis G, Velegraki A, Alexopoulos EC, Chasapi V, Tsigonia A, Katsambas A. Distribution of Malassezia species in pityriasis versicolor and seborrhoeic dermatitis in Greece. Typing of the major pityriasis versicolor isolate M. globosa. $\mathrm{Br} J$ Dermatol. 2006;154(5):854-9.

26. Crespo-Erchiga V, Gomez-Moyano E, Crespo M. [Pityriasis versicolor and the yeasts of genus Malassezia]. Actas Dermosifiliogr. 2008;99(10):764-71. 